\title{
Fine needle aspiration cytology as a preliminary diagnostic tool in chondroid syringoma: a case report and review
}

This article was published in the following Dove Press journal: Clinical, Cosmetic and Investigational Dermatology

\author{
MM Aarif Syed (D' \\ Upama Paudel (D) \\ Aasiya Rajbhandari $\mathbb{D}^{2}$ \\ Dinesh Binod Pokhrel (D) \\ Ram Chandra Adhikari $\mathbb{D}^{2}$ \\ Sudip Parajuli (D) \\ 'Department of Dermatology and \\ Venereology, Institute of Medicine, \\ Tribhuvan University, Kathmandu, Nepal; \\ ${ }^{2}$ Department of Pathology, Institute of \\ Medicine, Tribhuvan University, \\ Kathmandu, Nepal
}

\begin{abstract}
We report a case of chondroid syringoma (CS) in a 44-year-old male. He presented with a firm asymptomatic nodule in his left upper lip of 2-year duration. The initial clue to the diagnosis was made on fine needle aspiration cytology (FNAC), and a final diagnosis was based on histopathological examination. The case highlights the importance of FNAC in providing clues to the diagnosis of suspected cases of chondroid syringoma before performing large excisions and repair, which would require more skill and time. We have also reviewed the cytological findings of all the cases of benign CS reported until the current date.
\end{abstract}

Keywords: chondroid syringoma, pleomorphic adenoma, adnexal tumours, fine needle aspiration cytology

\section{Introduction}

Chondroid syringoma (CS) is a rare cutaneous tumour originating from eccrine and apocrine sweat glands with both epithelial and mesenchymal components. The incidence is low, forming less than $0.01 \%$ of primary cutaneous tumours. ${ }^{1}$ The clinical diagnosis is challenging. Histopathology is imperative to reach the diagnosis. The role of fine needle aspiration cytology (FNAC) has been underused in the past and is infrequently utilized for diagnosis or preoperative assessment. We report a case of benign chondroid syringoma of the upper lip, initially diagnosed by FNAC and later confirmed by histopathology. A literature review on the topic has also been done. The key words "chondroid syringoma" and "pleomorphic adenoma" were used to search databases which included PubMed, Google Scholar, Cochrane library and Hinari, and relevant papers were retrieved.

\section{Case presentation}

A 44-year-old male presented with an asymptomatic, progressive swelling over the left half of his upper lip of 2-years duration. The swelling was initially small and had gradually increased to present dimensions. There was no history of trauma, discharge or any other similar lesion in the body. There was no history of symptoms suggestive of systemic illnesses. On examination, there was a firm, non-fluctuant, skin coloured, non-tender nodule of size $1.5 \mathrm{~cm} \times 1.5 \mathrm{~cm}$ with overlying normal skin (Figure 1). The nodule was fixed to the skin, but freely mobile over underlying structures. There was no regional lymphadenopathy.
Correspondence: MM Aarif Syed Department of Dermatology and Venereology, Institute of Medicine,

Tribhuvan University, Maharajgunj Medical Campus, Maharajgunj, Kathmandu, 44600, Nepal

Tel +9 77I 44I 2707 (Ext 3052)

Email syedmmaarif@gmail.com 


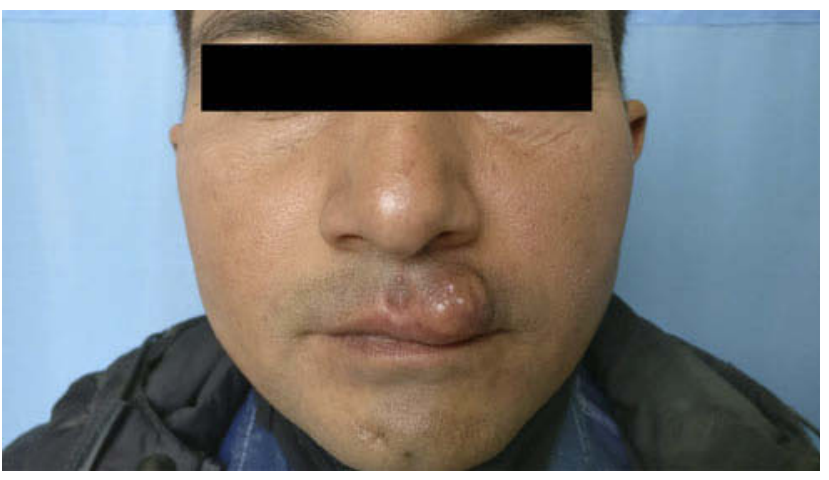

Figure I A $1.5 \mathrm{~cm}$ diameter nodule on left half of upper lip before excison.

FNAC of the lesion was done which showed aggregates, acini and singly scattered benign epithelial cells along with myoepithelial cells and chondromyxoid stromal fragments

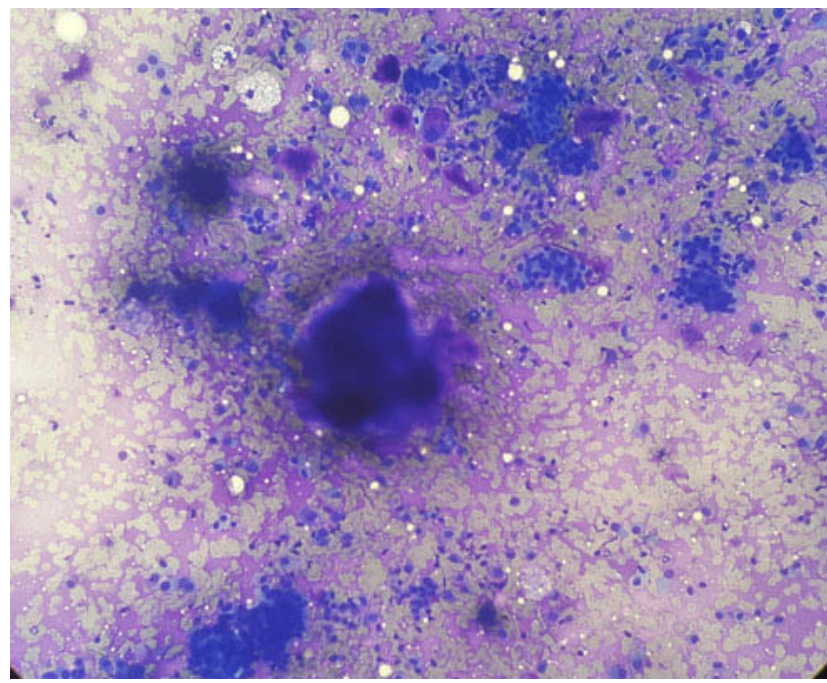

Figure 2 FNAC: aggregates, acini and single scattered benign epithelial cells along with myoepithelial cells and chondromyxoid stromal fragments (Giemsa stain, $\times 40$ ). Abbreviations: FNAC, fine needle aspiration cytology.
(Figure 2). Epithelial cells were round to polygonal with basophilic dense moderate cytoplasm and central to eccentric, round to oval nuclei with bland chromatin on a background of myxoid material, thus pointing towards the possibility of chondroid syringoma (Figure $3 \mathrm{~A}$ and $\mathrm{B}$ ).

The nodule was excised and the whole specimen was sent for histopathological examination. The cut section showed homogenous grey white areas. The hematoxylin-eosin stain revealed cystic structures with cystically dilated ducts, nests and glandular structures lined by bland looking epithelial cells along with surrounding chondromyxoid stroma (Figure 4A and B). Histopathology confirmed the cytological diagnosis of CS. Immunohistochemistry could not be done because of unavailability in the centre. Excision site was healthy during the postoperative period and no recurrence was observed after 6 months of follow-up.

\section{Discussion}

$\mathrm{CS}$ is a benign cutaneous tumor with male preponderance (male to female ratio of 5:8), ${ }^{2-5}$ and is predominantly seen on head and neck regions with predilection for upper lips, nose and cheeks. Rare sites on the face include the orbit, eyelids, and medial canthus. ${ }^{6-9}$ Other uncommon sites are the back, axilla, thighs, extremities and genitalia. ${ }^{2,3,5}$ The tumor presents as an asymptomatic, solitary, skin coloured, firm, and non-tender slow growing nodule. A tumor in the orbit may lead to exophthalmos. ${ }^{8}$ The size ranges from $0.3 \mathrm{~cm}$ to $3 \mathrm{~cm}$. Nodules exceeding $5 \mathrm{~cm}^{10}$ and $10 \mathrm{~cm}^{11,12}$ in diameter have also been reported. The clinical profiles of CS from five large retrospective studies are compared in Table 1.

\section{A}
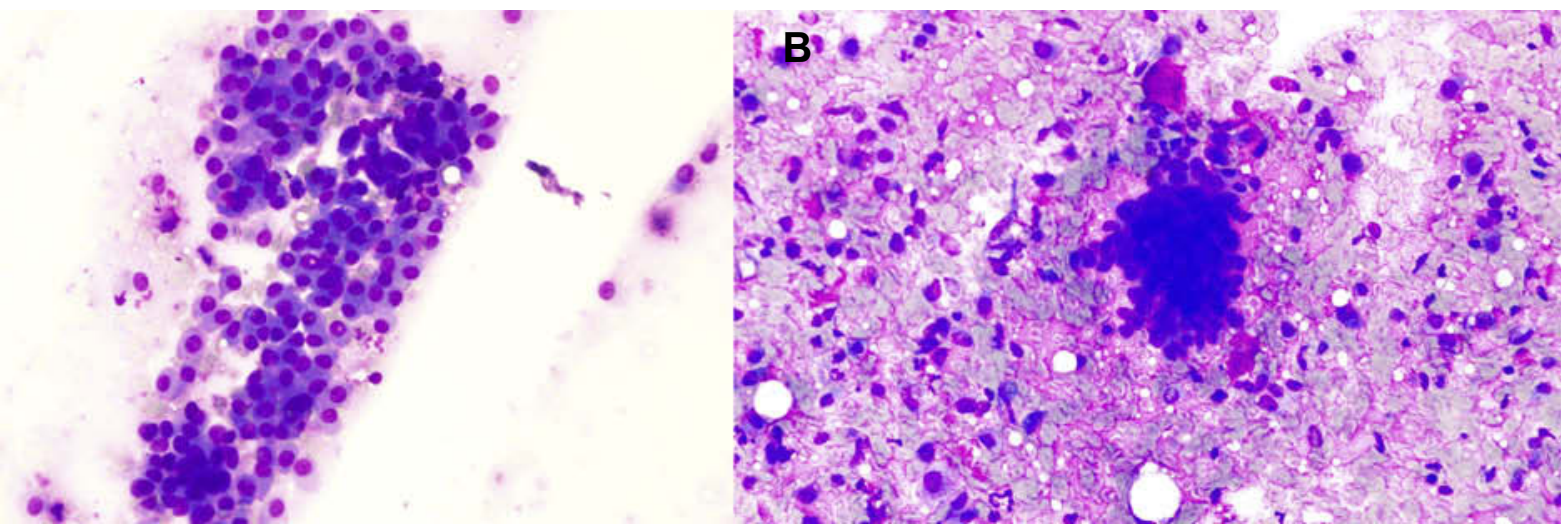

Figure 3 (A) FNAC smear showing sheet of myoepithelial cells with basophilic dense cytoplasm and central to eccentric, round to oval nuclei with bland chromatin (Giemsa stain, $\times 200)$. (B) Cluster of epithelial cells with scattered myoepithelial cells in a chondromyxoid background (Giemsa stain, $\times 200)$.

Abbreviations: FNAC, fine needle aspiration cytology. 


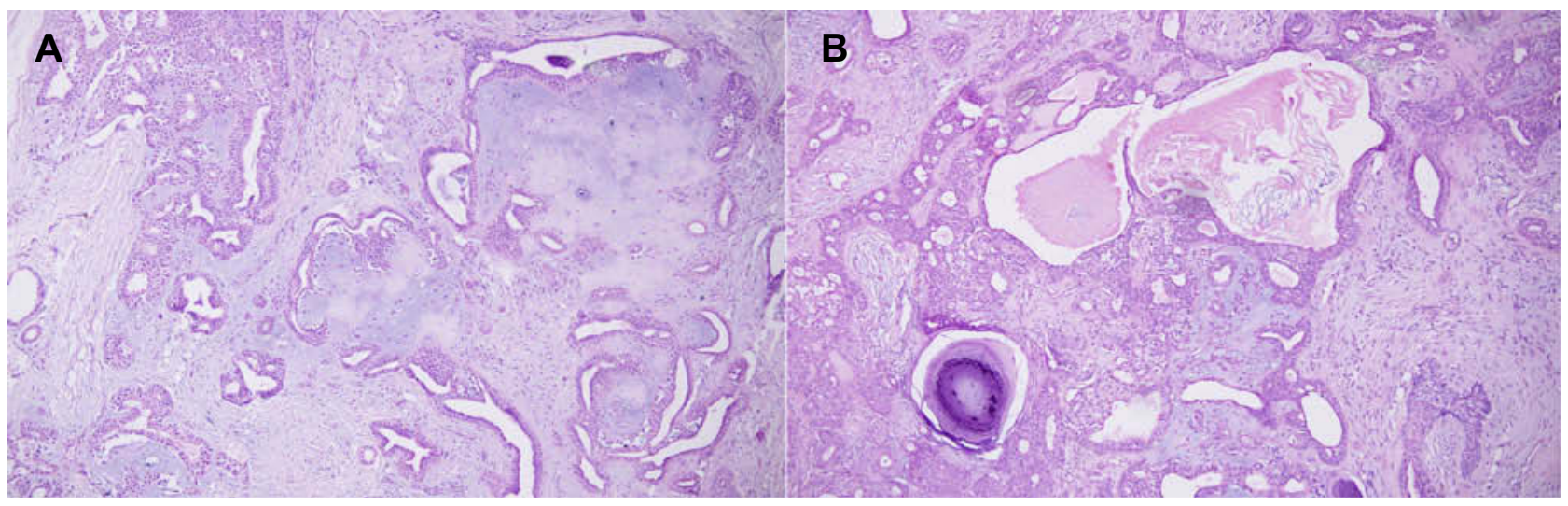

Figure 4 (A) Section showing tumor composed of ducts and glandular structures lined by bland looking epithelial and myoepithelial cells with surrounding chondromyxoid stroma (H\&E stain, $\times 40)$. (B) Focal areas showing ossification and keratinous cyst filled with keratin (H\&E stain, $\times 40)$.

Abbreviations: H\&E, hematoxylin and eosin.

Because of its rarity, asymptomatic and subcutaneous nature, the clinical diagnosis of this condition is often missed as evidenced by the diagnoses made before histopathological examination revealed in Table 2 . The differential diagnosis of such presentations include dermoid or sebaceous cyst, pilar cyst, calcifying epithelioma, or a solitary trichoepithelioma, dermatofibroma, lymph node, hamartoma, basal cell carcinoma, and seborrheic keratosis, ${ }^{4}$ with no role of non-invasive investigations like X-ray, ${ }^{14,15}$ ultrasonography, ${ }^{11,15,16} \mathrm{MRI},{ }^{10}$ or $\mathrm{CT} \operatorname{scan}^{8}$ in the diagnosis. FNAC and biopsy so far remains the gold standard for the diagnosis. FNAC which is easy to perform, is established in the literature for making early diagnosis of CS and is reviewed in Tables 2 and 3.

The origin of CS is from both secretory and ductal segments of eccrine or apocrine sweat glands. It is a mixed tumor with epithelial and mesenchymal components and resembles the pleomorphic adenoma of salivary glands. The first attempt to diagnose and document CS on FNAC was made in 1988 by Masood et al. ${ }^{17}$ The aspirate can be thick, mucoid and sometimes gelatinous with moderate cellularity. Thin aspirate may be associated with scanty stromal elements. ${ }^{18}$ The mucoid material stains positive with alcian blue and mucicarmine. ${ }^{17,19}$ The epithelial cells arrangement is highly variable. The cells can appear singly, scattered, in groups or as sheets, attached either loosely or cohesively. ${ }^{12,14,17,20-22}$ As in our case, acini ${ }^{9,19}$ and papillary ${ }^{16,23}$ configurations have also been noted. The individual cells are small to medium sized, well-defined, monomorphic, round-to-oval-to-ovoid-to-polygonal, with moderate to dense cytoplasm. The cytoplasm can be eosinophil to amphophilic, imparting a plasmacytoid appearance. ${ }^{20}$ The nuclei are small, monomorphic, round, oval, ovoid or elongated, central to eccentric in location with fine, evenly distributed chromatin. ${ }^{15,17,20,21,24}$ Nuclear atypia is a rare finding without any propensity to develop into malignancy. ${ }^{25}$ Anisonucleosis, conspicuous nucleoli or nuclei with clear halos may be suggestive of neoplastic changes, but malignancy can be safely ruled out in the absence of other features. ${ }^{14}$ The background is chondroid, ${ }^{17,22}$ myxoid, ${ }^{19,20,24}$ or chondromyxoid ${ }^{15,16,21,26-28}$ which can be scant $^{18}$ to abundant. ${ }^{29}$ Myoepithelial cells also appear in clusters or aggregates, dispersed along with epithelial cells in the stroma, and give plasmacytoid appearance with dark nuclei. ${ }^{21,23,29}$ Macrophages are uncommonly seen and have been reported along with cystic changes by Khan et al. ${ }^{18}$ In our case, foamy macrophages were evident on a myxoid background but without any cystic changes. Immunostaining differentiates the two components, as epithelial membrane antigen (EMA) and cytokeratin stains the epithelial cells, while S-100 makes the myoepithelial part evident. ${ }^{14,17}$

Cytology in addition to clinical features like site and size, can be a tool to differentiate benign from malignant $\mathrm{CS}^{30}$ Rarely, a benign tumor may turn aggressive and go into malignant phase. ${ }^{31}$ Poor prognosis, metastasis and recurrences following excision are attributes of malignant $\mathrm{CS}^{32-35}$ The clinical features that differentiates it from benign CS include female preponderance, predilection for extremities and size exceeding $3 \mathrm{~cm} .{ }^{17,30,36,37}$ Studies describing FNAC findings of malignant CS are also scarce. In 1997, Mishra et al ${ }^{36}$ made the first conclusive diagnosis of malignant CS on FNAC. Haemorrhagic aspirate, hypercellularity, pleomorphic epithelium, dyshesiveness of cells, intranuclear and intracytoplasmic vacuolation, and pericellular halo were the characteristic findings. Histopathology confirmed the diagnosis. A recent attempt to diagnose malignant CS on a recurrent lesion by FNAC was made in 2016 by Shobhanaa et al. ${ }^{38}$ The cytology 


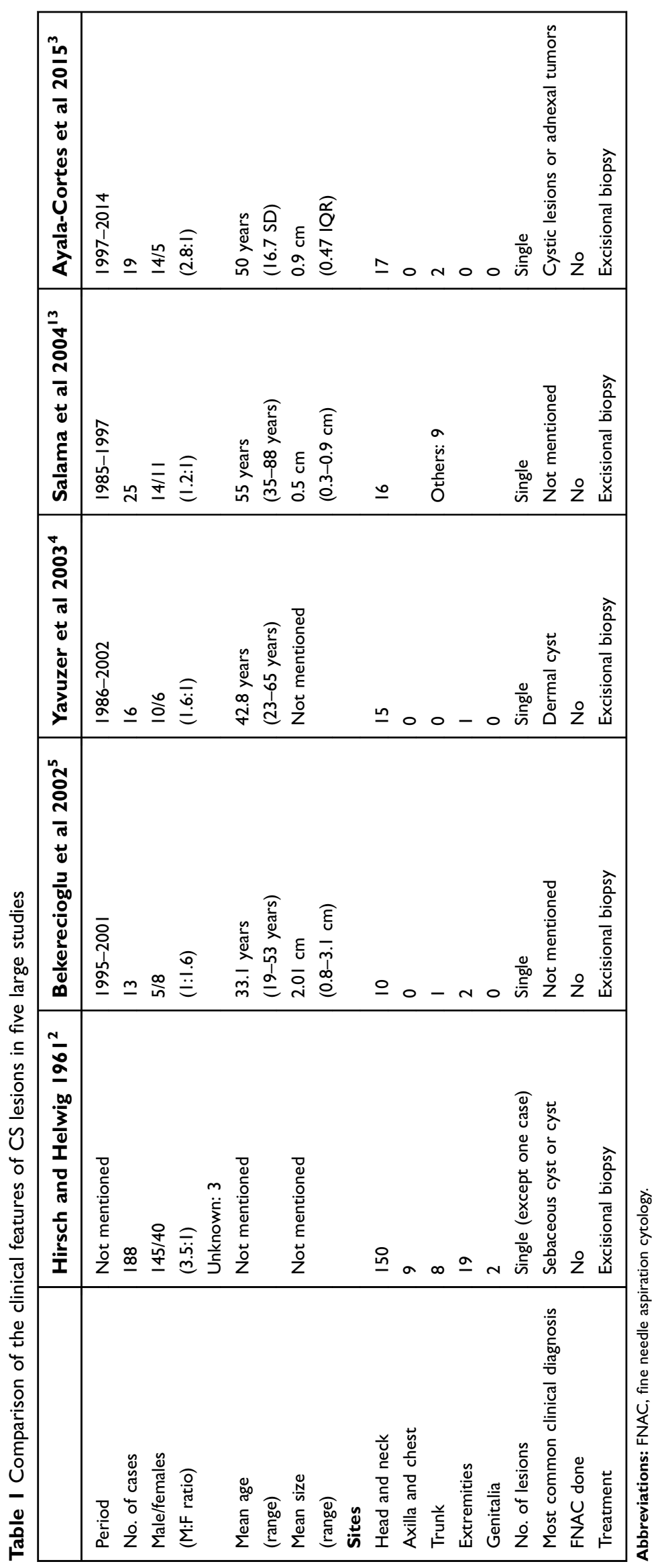


Table 2 Clinical findings and original diagnoses of cases which underwent FNAC

\begin{tabular}{|c|c|c|c|c|c|c|c|}
\hline & $\begin{array}{l}\text { Age } \\
\text { (years)/ } \\
\text { gender }\end{array}$ & Site & Size $(\mathrm{cm})$ & $\begin{array}{l}\text { Duration } \\
\text { (years) }\end{array}$ & $\begin{array}{l}\text { Clinical } \\
\text { diagnosis }\end{array}$ & $\begin{array}{l}\text { Cytological } \\
\text { diagnosis }\end{array}$ & $\begin{array}{l}\text { Histopathological } \\
\text { diagnosis }\end{array}$ \\
\hline $\begin{array}{l}\text { Masood } \\
\text { et al } 1988^{17}\end{array}$ & $76 / F$ & Left thigh & $5 \times 4$ & 5 & NM & CS & CS \\
\hline $\begin{array}{l}\text { Srinivasan } \\
\text { et al } 1993^{22}\end{array}$ & $60 / M$ & $\begin{array}{l}\text { Right } \\
\text { shoulder }\end{array}$ & 2.5 & 3 months & Neurofibroma & CS & CS \\
\hline $\begin{array}{l}\text { Gottschalk- } \\
\text { Sabag et al } \\
1994^{19}\end{array}$ & $82 / F$ & Axilla & $0.5 \times 3$ & NM & $\begin{array}{l}\text { Metastatic } \\
\text { lymph node }\end{array}$ & Probable CS & CS \\
\hline $\begin{array}{l}\text { Kumar et al } \\
2003^{21}\end{array}$ & $32 / M$ & $\begin{array}{l}\text { Nape of the } \\
\text { neck }\end{array}$ & $5 \times 5 \times 3$ & 2 & Hamartoma & $\begin{array}{l}\text { Benign appendageal } \\
\text { tumor of the skin }\end{array}$ & CS \\
\hline $\begin{array}{l}\text { Siddaraju } \\
\text { et al } 2009^{14}\end{array}$ & $43 / F$ & $\begin{array}{l}\text { Dorsum of } \\
\text { the nose }\end{array}$ & $0.8 \times 0.8$ & I & $\begin{array}{l}\text { Basal cell } \\
\text { carcinoma }\end{array}$ & CS & CS \\
\hline $\begin{array}{l}\text { Kumar } \\
2010^{15}\end{array}$ & $20 / M$ & $\begin{array}{l}\text { Dorsum of } \\
\text { nose }\end{array}$ & $2 \times 2$ & NM & Dermoid cyst & CS & CS \\
\hline $\begin{array}{l}\text { Skoro et al } \\
2010^{16}\end{array}$ & $63 / M$ & Neck & 0.8 & 5 & NM & CS & CS \\
\hline Dubb et al & $32 / F$ & Scalp & 2 & NM & NM & Suggestive of CS & CS \\
\hline $2010^{20}$ & $\begin{array}{l}23 / \mathrm{M} \\
18 / \mathrm{F}\end{array}$ & $\begin{array}{l}\text { Scalp } \\
\text { Upper lip }\end{array}$ & $\begin{array}{l}2 \\
0.5\end{array}$ & & & & \\
\hline $\begin{array}{l}\text { Tokyol et al } \\
2010^{28}\end{array}$ & $57 / F$ & Philtrum & 1.5 & 10 & Lipoma & $\begin{array}{l}\text { Benign appendageal } \\
\text { tumor }\end{array}$ & CS \\
\hline $\begin{array}{l}\text { Nasit et al } \\
2012^{27}\end{array}$ & $40 / F$ & Mastoid & $1.2 \mathrm{~cm}$ & 3 & None & CS & CS \\
\hline $\begin{array}{l}\text { Narasimha } \\
\text { et al } 2013^{12}\end{array}$ & $50 / M$ & Lower back & $12 \times 8 \times 5$ & 3 & NM & CS & CS \\
\hline Khan $2013^{18}$ & $3 I / M$ & $\begin{array}{l}\text { Left } \\
\text { supraorbital } \\
\text { region }\end{array}$ & $3 \times 2.5$ & NM & $\begin{array}{l}\text { Sebaceous or } \\
\text { epidermal cyst }\end{array}$ & $\begin{array}{l}\text { Benign cystic neo- } \\
\text { plasm possibly } \\
\text { benign skin adnexal } \\
\text { tumor }\end{array}$ & CS \\
\hline $\begin{array}{l}\text { Pal et al } \\
2014^{29}\end{array}$ & $33 / M$ & Left forearm & $2 \times 1.5$ & $11 / 2$ & NM & CS & CS \\
\hline $\begin{array}{l}\text { Barman et al } \\
2016^{39}\end{array}$ & $25 / M$ & Right thumb & 3.5 & 2 & NM & NM & CS \\
\hline $\begin{array}{l}\text { Rogers et al } \\
2016^{24}\end{array}$ & $67 / M$ & Right axilla & I & I & $\begin{array}{l}\text { Lymph node or } \\
\text { cyst }\end{array}$ & $\begin{array}{l}\text { Benign epithelial- } \\
\text { mesenchymal bipha- } \\
\text { sic neoplasm }\end{array}$ & CS \\
\hline $\begin{array}{l}\text { Mahantappa } \\
\text { et al } 2016^{23}\end{array}$ & $40 / M$ & $\begin{array}{l}\text { Anterior } \\
\text { abdominal } \\
\text { wall }\end{array}$ & $8 \times 6 \times 5$ & $11 / 2$ & Dermoid cyst & CS & CS \\
\hline $\begin{array}{l}\text { Lamba et al } \\
2017^{26}\end{array}$ & $37 / M$ & Left arm & $2.5 \times 2$ & I & $\begin{array}{l}\text { Epidermal inclu- } \\
\text { sion cyst }\end{array}$ & CS & CS \\
\hline $\begin{array}{l}\text { Our case } \\
2018\end{array}$ & $44 / M$ & Upper lip & $1.5 \times 1.5$ & 6 & $\begin{array}{l}\text { Sebaceous cyst } \\
\text { and dermatofi- } \\
\text { broma }\end{array}$ & CS & CS \\
\hline
\end{tabular}

Abbreviations: CS, chondroid syringoma; FNAC, fine needle aspiration cytology; NM, Not mentioned. 
Table 3 Detail cytological findings of cases which underwent FNAC

\begin{tabular}{|c|c|c|c|c|c|c|c|}
\hline & Aspirate & Cellularity & $\begin{array}{l}\text { Epithelial } \\
\text { cell } \\
\text { arrangement }\end{array}$ & $\begin{array}{l}\text { Individual } \\
\text { cells }\end{array}$ & Nuclei & $\begin{array}{l}\text { Myoepitheliod } \\
\text { cells }\end{array}$ & $\begin{array}{l}\text { Background/ } \\
\text { Stroma }\end{array}$ \\
\hline $\begin{array}{l}\text { Masood } \\
\text { et al } 1988^{17}\end{array}$ & $N M$ & Moderate & $\begin{array}{l}\text { Clusters and } \\
\text { sheets }\end{array}$ & $\begin{array}{l}\text { Small cells with } \\
\text { relatively scant, } \\
\text { faintly eosinophi- } \\
\text { lic cytoplasm }\end{array}$ & $\begin{array}{l}\text { Small ovoid-to- } \\
\text { elongated with } \\
\text { finely granular } \\
\text { chromatin, } \\
\text { occasional } \\
\text { small chromo- } \\
\text { centers }\end{array}$ & NM & Chondroid \\
\hline $\begin{array}{l}\text { Srinivasan } \\
\text { et al } 1993^{22}\end{array}$ & NM & NM & NM & $\begin{array}{l}\text { Round to oval } \\
\text { cells with } \\
\text { a moderate } \\
\text { amount of } \\
\text { cytoplasm }\end{array}$ & $\begin{array}{l}\text { Monomorphic } \\
\text { nuclei }\end{array}$ & $\begin{array}{l}\text { Some spindle- } \\
\text { shaped cells }\end{array}$ & $\begin{array}{l}\text { Myxoid } \\
\text { (Abundant) }\end{array}$ \\
\hline $\begin{array}{l}\text { Gottschalk- } \\
\text { Sabag et al } \\
1994^{19}\end{array}$ & NM & NM & $\begin{array}{l}\text { Single, groups } \\
\text { and tubular } \\
\text { configuration }\end{array}$ & Regular & NM & NM & Myxoid \\
\hline $\begin{array}{l}\text { Kumar et al } \\
2003^{21}\end{array}$ & $\begin{array}{l}\text { Thick, } \\
\text { mucoid } \\
\text { and } \\
\text { gelatinous }\end{array}$ & & $\begin{array}{l}\text { Clusters of } \\
\text { epithelial }\end{array}$ & $\begin{array}{l}\text { Round and } \\
\text { monomorphous } \\
\text { with moderate to } \\
\text { abundant amount } \\
\text { of cytoplasm }\end{array}$ & $\begin{array}{l}\text { Monomorphic, } \\
\text { with fine chro- } \\
\text { matin Some } \\
\text { eccentrically } \\
\text { placed }\end{array}$ & In clusters & $\begin{array}{l}\text { Metachromatic, } \\
\text { chondromyxoid }\end{array}$ \\
\hline $\begin{array}{l}\text { Siddaraju } \\
\text { et al } 2009^{14}\end{array}$ & NM & Moderate & $\begin{array}{l}\text { Clusters as well } \\
\text { as dispersed }\end{array}$ & $\begin{array}{l}\text { Round to poly- } \\
\text { gonal with mod- } \\
\text { erate to } \\
\text { abundant cyto- } \\
\text { plasm } \\
\text { A few occasional, } \\
\text { tiny clusters of } \\
\text { bland spindle } \\
\text { cells }\end{array}$ & $\begin{array}{l}\text { Oval, vesicular } \\
\text { with mild to } \\
\text { moderate ani- } \\
\text { sonucleosis } \\
\text { Some with } \\
\text { conspicuous } \\
\text { nucleoli and } \\
\text { chromocenters } \\
\text { Occasional } \\
\text { rounded nuclei } \\
\text { with clear halos }\end{array}$ & NM & $\begin{array}{l}\text { Relatively pale- } \\
\text { stained, cyano- } \\
\text { philic to eosino- } \\
\text { philic ground } \\
\text { substance }\end{array}$ \\
\hline $\begin{array}{l}\text { Kumar } \\
2010^{15}\end{array}$ & Mucoid & NM & 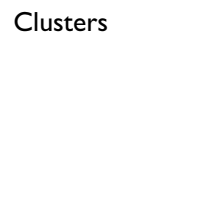 & $\begin{array}{l}\text { Round with } \\
\text { moderate to } \\
\text { abundant } \\
\text { cytoplasm }\end{array}$ & $\begin{array}{l}\text { Monomorphic, } \\
\text { centrally to } \\
\text { eccentrically } \\
\text { located } \\
\text { Fine chromatin }\end{array}$ & NM & Chondromyxoid \\
\hline $\begin{array}{l}\text { Skoro et al } \\
2010^{16}\end{array}$ & Bloody & NM & $\begin{array}{l}\text { Clusters and } \\
\text { papillary } \\
\text { formations }\end{array}$ & $\begin{array}{l}\text { Well defined with } \\
\text { dense, moderate } \\
\text { cytoplasm }\end{array}$ & $\begin{array}{l}\text { Round to } \\
\text { ovale, centrally } \\
\text { located } \\
\text { Fine, evenly } \\
\text { distributed } \\
\text { chromatin }\end{array}$ & NM & Chondromyxoid \\
\hline
\end{tabular}


Table 3 (Continued).

\begin{tabular}{|c|c|c|c|c|c|c|c|}
\hline & Aspirate & Cellularity & $\begin{array}{l}\text { Epithelial } \\
\text { cell } \\
\text { arrangement }\end{array}$ & $\begin{array}{l}\text { Individual } \\
\text { cells }\end{array}$ & Nuclei & $\begin{array}{l}\text { Myoepitheliod } \\
\text { cells }\end{array}$ & $\begin{array}{l}\text { Background/ } \\
\text { Stroma }\end{array}$ \\
\hline $\begin{array}{l}\text { Dubb et al } \\
2010^{20}\end{array}$ & NM & NM & $\begin{array}{l}\text { Sheets, clusters } \\
\text { and single cells }\end{array}$ & $\begin{array}{l}\text { Well defined with } \\
\text { moderate eosi- } \\
\text { nophilic to } \\
\text { amphophilic } \\
\text { cytoplasm } \\
\text { imparting } \\
\text { a plasmacytoid } \\
\text { appearance }\end{array}$ & $\begin{array}{l}\text { Bland, round to } \\
\text { oval, eccentri- } \\
\text { cally located } \\
\text { Evenly dis- } \\
\text { persed, hypo- } \\
\text { chromatic } \\
\text { chromatin } \\
\text { Small, incon- } \\
\text { spicuous } \\
\text { nucleoli. }\end{array}$ & NM & $\begin{array}{l}\text { Eosinophilic } \\
\text { myxoid }\end{array}$ \\
\hline $\begin{array}{l}\text { Tokyol et al } \\
2010^{28}\end{array}$ & NM & Hypercellular & $\begin{array}{l}\text { Cohesive groups } \\
\text { of cells }\end{array}$ & $\begin{array}{l}\text { Monomorphic } \\
\text { round cells with } \\
\text { moderate to } \\
\text { abundant amount } \\
\text { cytoplasm }\end{array}$ & $\begin{array}{l}\text { Monomorphic } \\
\text { nuclei with fine } \\
\text { chromatin. } \\
\text { Some nuclei } \\
\text { were eccentri- } \\
\text { cally placed, } \\
\text { like plasmacy- } \\
\text { toid cells }\end{array}$ & Spindle cells seen & Chondromyxoid \\
\hline $\begin{array}{l}\text { Nasit et al } \\
2012^{27}\end{array}$ & $\begin{array}{l}\text { Thick and } \\
\text { mucoid }\end{array}$ & NM & $\begin{array}{l}\text { Sheets and loose } \\
\text { clusters with } \\
\text { a few single cells }\end{array}$ & $\begin{array}{l}\text { Bland, small and } \\
\text { monomorphic } \\
\text { with moderate } \\
\text { amount of } \\
\text { cytoplasm }\end{array}$ & $\begin{array}{l}\text { Round to-oval, } \\
\text { centrally } \\
\text { located Evenly } \\
\text { dispersed fine } \\
\text { chromatin }\end{array}$ & Elongated & Chondromyxoid \\
\hline $\begin{array}{l}\text { Narasimha } \\
\text { et al } 2013^{12}\end{array}$ & $\begin{array}{l}\text { Thick, } \\
\text { mucoid, } \\
\text { and } \\
\text { gelatinous }\end{array}$ & NM & $\begin{array}{l}\text { Loose cohesive } \\
\text { clusters and } \\
\text { discretes }\end{array}$ & $\begin{array}{l}\text { Round to oval } \\
\text { with moderate to } \\
\text { abundant } \\
\text { cytoplasm }\end{array}$ & $\begin{array}{l}\text { Centrally } \\
\text { located nuclei } \\
\text { having fine } \\
\text { chromatin, } \\
\text { a few showing } \\
\text { one to two } \\
\text { prominent } \\
\text { nucleoli }\end{array}$ & NM & Chondromyxoid \\
\hline Khan $2013^{18}$ & $\begin{array}{l}\text { Thin fluid- } \\
\text { like }\end{array}$ & 更 & $\begin{array}{l}\text { Cohesive clus- } \\
\text { ters } \\
\text { A few acinar } \\
\text { formation }\end{array}$ & $\begin{array}{l}\text { Medium-sized } \\
\text { cells with moder- } \\
\text { ate to abundant } \\
\text { amount of } \\
\text { cytoplasm }\end{array}$ & $\begin{array}{l}\text { Bland appearing } \\
\text { monomorphic } \\
\text { centrally placed } \\
\text { or slightly } \\
\text { eccentric nuclei } \\
\text { with fine } \\
\text { chromatin }\end{array}$ & $\begin{array}{l}\text { Smaller } \\
\text { hyperchromatic }\end{array}$ & $\begin{array}{l}\text { Chondromyxoid } \\
\text { (Scant) }\end{array}$ \\
\hline $\begin{array}{l}\text { Pal et al } \\
2014^{29}\end{array}$ & $\begin{array}{l}\text { Thick } \\
\text { mucoid }\end{array}$ & Moderate & Clusters & $\begin{array}{l}\text { Monomorphic, } \\
\text { round to oval, } \\
\text { medium sized } \\
\text { having moderate } \\
\text { amount of } \\
\text { cytoplasm }\end{array}$ & $\begin{array}{l}\text { Bland round to } \\
\text { oval with finely } \\
\text { dispersed } \\
\text { chromatin }\end{array}$ & $\begin{array}{l}\text { Small cells having } \\
\text { plasmacytoid } \\
\text { appearance with } \\
\text { dark nuclei }\end{array}$ & $\begin{array}{l}\text { Chondromyxoid } \\
\text { (Abundant) }\end{array}$ \\
\hline
\end{tabular}




\begin{tabular}{|c|c|c|c|c|c|c|c|}
\hline & Aspirate & Cellularity & $\begin{array}{l}\text { Epithelial } \\
\text { cell } \\
\text { arrangement }\end{array}$ & $\begin{array}{l}\text { Individual } \\
\text { cells }\end{array}$ & Nuclei & $\begin{array}{l}\text { Myoepitheliod } \\
\text { cells }\end{array}$ & $\begin{array}{l}\text { Background/ } \\
\text { Stroma }\end{array}$ \\
\hline $\begin{array}{l}\text { Barman et al } \\
2016^{39}\end{array}$ & NM & NM & $\begin{array}{l}\text { Loose clusters } \\
\text { and sheets }\end{array}$ & $\begin{array}{l}\text { Ovoid and spin- } \\
\text { dle Cells with } \\
\text { moderate to the } \\
\text { abundant well-- } \\
\text { defined } \\
\text { cytoplasm }\end{array}$ & $\begin{array}{l}\text { Oval with bland } \\
\text { finely granular } \\
\text { chromatin }\end{array}$ & NM & Chondromyxoid \\
\hline $\begin{array}{l}\text { Rogers et al } \\
2016^{24}\end{array}$ & NM & Moderate & $\begin{array}{l}\text { Loose or } \\
\text { clusters }\end{array}$ & $\begin{array}{l}\text { Epithelioid to } \\
\text { spindled with } \\
\text { a moderate } \\
\text { amount of } \\
\text { cytoplasm }\end{array}$ & $\begin{array}{l}\text { Round to ovoid } \\
\text { nuclei, and } \\
\text { inconspicuous } \\
\text { nucleoli }\end{array}$ & & Myxoid \\
\hline $\begin{array}{l}\text { Mahantappa } \\
\text { et al } 2016^{23}\end{array}$ & $\begin{array}{l}\text { Scant and } \\
\text { Mucoid }\end{array}$ & Scant & $\begin{array}{l}\text { Clusters, groups, } \\
\text { in papillae }\end{array}$ & $\begin{array}{l}\text { Small to medium } \\
\text { size with well- } \\
\text { defined cell bor- } \\
\text { ders having scant- } \\
\text { to-moderate } \\
\text { amounts of } \\
\text { cytoplasm }\end{array}$ & $\begin{array}{l}\text { Round to oval } \\
\text { with fine } \\
\text { stippled } \\
\text { chromatin }\end{array}$ & Clusters & Myxochondroid \\
\hline $\begin{array}{l}\text { Lamba et al } \\
2017^{26}\end{array}$ & $\begin{array}{l}\text { Thick } \\
\text { mucoid }\end{array}$ & NM & NM & $\begin{array}{l}\text { Monomorphic, } \\
\text { round to oval, } \\
\text { with moderate } \\
\text { amount of } \\
\text { cytoplasm }\end{array}$ & $\begin{array}{l}\text { Centrally } \\
\text { placed nuclei } \\
\text { with fine } \\
\text { chromatin }\end{array}$ & NM & Chondromyxoid \\
\hline $\begin{array}{l}\text { Our case } \\
2018\end{array}$ & $\begin{array}{l}\text { Blood } \\
\text { mixed }\end{array}$ & & $\begin{array}{l}\text { Aggregates, acini } \\
\text { and singly } \\
\text { scattered }\end{array}$ & $\begin{array}{l}\text { Round to poly- } \\
\text { gonal with baso- } \\
\text { philic dense } \\
\text { moderate } \\
\text { cytoplasm }\end{array}$ & $\begin{array}{l}\text { Central to } \\
\text { eccentric, } \\
\text { round to oval } \\
\text { nuclei with } \\
\text { bland } \\
\text { chromatin }\end{array}$ & Aggregates & Chondromyxoid \\
\hline
\end{tabular}

Abbreviations: NM, not mentioned.

revealed hypercellularity and tissue fragments of malignantappearing round-to-polygonal cells. The biopsy was inconclusive. A repeat FNAC was performed, along with immunocytochemistry. Vacuolation, indistinct cell borders, nuclear pleomorphism and multiple prominent nucleoli were appreciated. Pan cytokeratin, EMA, S-100, calponin, and $\alpha$-smooth muscle actin showed strong positivity, which sealed the diagnosis.

Excision is the treatment of choice and should include the margins. In 1961, Hirsch and Helwig ${ }^{2}$ proposed the histological criteria for diagnosis of CS. Apocrine CS exceeds the number of eccrine CS reported. The apocrine variant has two rows of epithelial cells lining the tubular and cystic branching lumina, while the smaller lumen in eccrine type has a single row of cells. ${ }^{4}$ The presented case belonged to the former group.

\section{Conclusion}

CS is a rare tumour presenting in dermatological practice. FNAC is a very useful tool for making preliminary diagnosis of CS before making a large excision. However, the final diagnosis is based on histopathological examination.

\section{Ethical statement}

The patient gave his written informed consent for the publication of images and information. Institutional approval was not required to publish the case details. 


\section{Disclosure}

The authors report no conflicts of interest in this work.

\section{References}

1. Tural D, Selcukbiricik F, Gunver F, et al. Facial localization of malignant chondroid syringoma: a rare case report. Case Rep Oncol Med. 2013;2013:3. doi:10.1155/2013/907980

2. Hirsch P, Helwig EB. Chondroid syringoma. Mixed tumor of skin, salivary gland type. Arch Dermatol. 1961;84:835-847.

3. Ayala-Cortes AS, Martinez-Cabriales SA, Vazquez-Martinez O, et al. Chondroid syringoma: a challenging clinical diagnosis. $J$ Am Acad Dermatol. 2015;72(5):AB42. doi:10.1016/j.jaad.2015.02.178

4. Yavuzer R, Basterzi Y, Sari A, Bir F, Sezer C. Chondroid syringoma: a diagnosis more frequent than expected. Dermatol Surg. 2003;29 (2):179-181.

5. Bekerecioglu M, Tercan M, Karakok M, Atik B. Benign chondroid syringoma: a confusing clinical diagnosis. Eur J Plast Surg. 2002;25 (6):316-318. doi:10.1007/s00238-002-0385-5

6. Paraskevopoulos K, Cheva A, Koloutsos G, Matzarakis I, Vahtsevanos K. Chondroid syringoma of the medial canthus. Case Rep Otolaryngol. 2014;2014:158527. doi:10.1155/2014/158527

7. Kitazawa T, Hataya Y, Matsuo K. Chondroid syringoma of the orbit. Ann Plast Surg. 1999;42(1):100-102.

8. Belfquih H, El Mostarchid B, Oukabli M, Akhaddar A, Boucetta M. Benign chondroid syringoma of the orbit: a rare cause of exophtalmos. Head Face Med. 2012;8:8. doi:10.1186/1746-160X-8-8

9. Kumar MA, Srikanth K, Vathsalya R. Chondroid syringoma: a rare lid tumor. Indian J Ophthalmol. 2013;61(1):43-44. doi:10.4103/03014738.105060

10. Uyar B, Solak A, Sahin N, Bugdayci H. Giant chondroid syringoma radiologically mimicking malignancy. Indian J Dermatol. 2013;58 (3):245. doi:10.4103/0019-5154.110879

11. Sungur N, Uysal A, Gumus M, Kocer U. An unusual chondroid syringoma. Dermatol Surg. 2003;29(9):977-979.

12. Narasimha A, Kalyani R, Kumar HML, Suresh TN, Supreeth A. Giant chondroid syringoma with divergent differentiation: cyto-histoimmuno correlation. Int J Appl Basic Med Res. 2013;3(2):129-131. doi:10.4103/2229-516X.117097

13. Salama ME, Azam M, Ma CK, et al. Chondroid syringoma. Cytokeratin 20 immunolocalization of Merkel cells and reappraisal of apocrine folliculo-sebaceous differentiation. Arch Pathol Lab Med. 2004;128 (9):986-990. doi:10.1043/1543-2165(2004)128<986:CS $>2.0$.CO;2

14. Siddaraju N, Murugan P, Wilfred CD, Choudhury N, Basu D. Preoperative cytologic diagnosis of chondroid syringoma. Acta Cytol. 2009;53(5):607-610. doi:10.1159/000325396

15. Kumar B. Chondroid syringoma diagnosed by fine needle aspiration cytology. Diagn Cytopathol. 2010;38(1):38-40. doi:10.1002/dc.21159

16. Skoro M, Ostovic KT, Cikara I, Muller D, Novak NP, Virag M. Fine needle aspiration cytology of chondroid syringoma. Coll Antropol. 2010;34(2):687-690.

17. Masood S, Hardy NM. Fine needle aspiration cytology of chondroid syringoma. Report of a case. Acta Cytol. 1988;32(4):482-484.

18. Khan K. Chondroid syringoma: a case with unusual cytological findings. Indian J Dermatol. 2013;58(2):157. doi:10.4103/0019-5154.108072

19. Gottschalk-Sabag S, Glick T. Chondroid syringoma diagnosed by fine-needle aspiration: a case report. Diagn Cytopathol. 1994;10 (2):152-155.

20. Dubb M, Michelow P. Cytologic features of chondroid syringoma in fine needle aspiration biopsies: a report of 3 cases. Acta Cytol. 2010;54(2):183-186. doi:10.1159/000325005
21. Kumar S, Ghotekar LH, Thappa DM, Smile R. Diagnosis of chondroid syringoma by fine needle aspiration cytology. Acta Cytol. 2003;47(3):522-524.

22. Srinivasan R, Rajwanshi A, Padmanabhan V, Dey P. Fine needle aspiration cytology of chondroid syringoma and syringocystadenoma papilliferum. A report of two cases. Acta Cytol. 1993;37(4):535-538.

23. Mahantappa H, Ravindra S, Ranganna R, Krishnamurthy $T$. Cytomorphology of giant chondroid syringoma of the abdominal wall: a case report. Arch Med Health Sci. 2016;4(1):116-118. doi:10.4103/2321-4848.183369

24. Rogers R, Zhou F, Grunes D, et al. Chondroid syringoma of the axilla: an unusual tumor diagnosed by fine needle aspiration. Diagn Cytopathol. 2016;44(4):342-346. doi:10.1002/dc.23424

25. Rege J, Shet T. Aspiration cytology in the diagnosis of primary tumors of skin adnexa. Acta Cytol. 2001;45(5):715-722. doi:10.1159/000328293

26. Lamba S, Nanda A, Kumar U. Chondroid syringoma: fine-needle aspiration cytology of a rare entity at an unusual site. J Clin Diagn Res. 2017;11(7):ED06-ED7. doi:10.7860/JCDR/2017/28405.10135

27. Nasit JG, Dhruva G. Chondroid syringoma: a diagnosis by fine needle aspiration cytology. J Cutan Aesthet Surg. 2012;5 (3):222-225. doi:10.4103/0974-2077.101404

28. Tokyol C, Aktepe F, Yavas BD, Yildiz H, Aycicek A. Chondroid syringoma: a case report. Acta Cytol. 2010;54(5 Suppl):973-976.

29. Pal S, Sengupta S, Jana S, Bose K. Fine-needle aspiration cytology of chondroid syringoma of fore arm: report of a rare case. J Cytol. 2014;31(3):171-173. doi:10.4103/0970-9371.145659

30. Redono C, Rocamora A, Villoria F, Garcia M. Malignant mixed tumor of the skin: malignant chondroid syringoma. Cancer. 1982;49 (8):1690-1696.

31. Shvili D, Rothem A. Fulminant metastasizing chondroid syringoma of the skin. Am J Dermatopathol. 1986;8(4):321-325.

32. Schiano di Visconte M, Picciano P. Chondroid syringoma. A case report. Chir Ital. 2002;54(2):241-244.

33. Barnett MD, Wallack MK, Zuretti A, Mesia L, Emery RS, Berson AM. Recurrent malignant chondroid syringoma of the foot: a case report and review of the literature. Am J Clin Oncol. 2000;23(3):227-232.

34. Kim DH, Lee CW, Lee KH, Hyun MS. A case of malignant chondroid syringoma with lung metastasis. Cancer Res Treat. 1997;29 (6): 1119 .

35. Watarai A, Amoh Y, Aki R, Takasu H, Katsuoka K. Malignant chondroid syringoma: report of a case with lymph node metastasis 12 years after local excision. Dermatol Online J. 2011;17 (9):5.

36. Mishra K, Agarwal S. Fine needle aspiration cytology of malignant chondroid syringoma: a case report. Acta Cytol. 1998;42 (5):1155-1158. doi:10.1159/000332105

37. Constantinescu MB, Chan JB, Cassarino DS. Chondroid syringoma with tyrosine crystals: case report and review of the literature. Am J Dermatopathol. 2010;32(2):171-174. doi:10.1097/DAD.0b013e3181aec131

38. Shobhanaa P, Siddaraju N, Jinkala S, Badhe B, Ganesh R. Emphasizing the pivotal role of fine-needle aspiration cytology in a case of recurrent malignant chondroid syringoma. J Cytol. 2016;33 (2):103-105. doi:10.4103/0970-9371.177148

39. Barman D, Bhowmik A. An unusual presentation of chondroid syringoma. Indian J Pathol Microbiol. 2016;59(3):362-364. doi:10.4103/0377-4929.188111 


\section{Publish your work in this journal}

Clinical, Cosmetic and Investigational Dermatology is an international, peer-reviewed, open access, online journal that focuses on the latest clinical and experimental research in all aspects of skin disease and cosmetic interventions. This journal is indexed on CAS.

The manuscript management system is completely online and includes a very quick and fair peer-review system, which is all easy to use. Visit http://www.dovepress.com/testimonials.php to read real quotes from published authors.

Submit your manuscript here: https://www.dovepress.com/clinical-cosmetic-and-investigational-dermatology-journal 